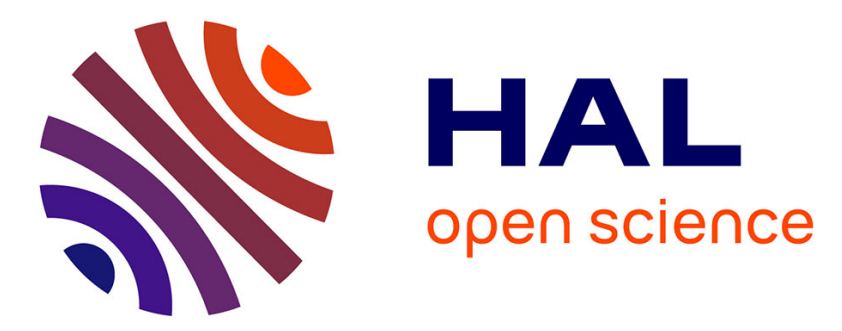

\title{
Influence d'une turbulence thermique sur la propagation du son dans l'atmosphère en présence d'une zone d'ombre
}

\author{
P. Chevret, Ph. Blanc-Benon
}

\section{- To cite this version:}

P. Chevret, Ph. Blanc-Benon. Influence d'une turbulence thermique sur la propagation du son dans l'atmosphère en présence d'une zone d'ombre. Journal de Physique IV Proceedings, 1994, 04 (C5), pp.C5-969-C5-972. 10.1051/jp4:19945212 . jpa-00252897

\section{HAL Id: jpa-00252897 https://hal.science/jpa-00252897}

Submitted on 1 Jan 1994

HAL is a multi-disciplinary open access archive for the deposit and dissemination of scientific research documents, whether they are published or not. The documents may come from teaching and research institutions in France or abroad, or from public or private research centers.
L'archive ouverte pluridisciplinaire HAL, est destinée au dépôt et à la diffusion de documents scientifiques de niveau recherche, publiés ou non, émanant des établissements d'enseignement et de recherche français ou étrangers, des laboratoires publics ou privés. 


\title{
Influence d'une turbulence thermique sur la propagation du son dans l'atmosphère en présence d'une zone d'ombre
}

\author{
P. CHEVRET et Ph. BLANC-BENON
}

Ecole Centrale de Lyon, Laboratoire de Mécanique des Fluides et d'Acoustique, URA 263 du CNRS, ECL, BP. 163, 69131 Ecully cedex, France

\begin{abstract}
A numerical technique for simulating the acoustic wave propagation through a turbulent atmosphere is introduced. We first generate a random homogeneous and isotropic medium by creating $\mathrm{N}$ independent realizations in terms of discrete Fourier modes. Then, for each realization, we solve a parabolic equation using a Split step Padé algorithm. Finally, we calculate the mean intensity of the acoustic field and its phase and amplitude fluctuations. Our numerical results are compared with outdoor measurements.
\end{abstract}

\section{INTRODUCTION}

La connaissance du comportement du champ acoustique dans l'atmosphère est un problème d'actualité puisque notre société s'oriente vers le contrôle des nuisances acoustiques à proximité d'aéroports dans les grandes villes ou d'installations industrielles, pour un meilleur confort auditif. Une grande quantité de mesures ont été réalisées en extérieur depuis les années 1950. Ces mesures mettent en évidence, pour la plupart, le rôle de la turbulence dans deux situations particulières de propagation.

- la propagation sur un sol rigide où les figures d'interférence issues de la superposition de l'onde directe et de l'onde réfléchie sur le sol, sont "gommées" par les inhomogénéités de la turbulence.

- la propagation dans une zone d'ombre, créée par un gradient de célérité négatif, dans laquelle les inhomogénéités diffusent l'énergie de telle sorte que les niveaux moyens sont plus élevés que ceux prédits par les théories déterministes.

Parallèlement à ces mesures, nous avons développé un code numérique pour la prédiction de la propagation $\mathrm{du}$ son dans une atmosphère turbulente. La réalisation de ce code passe par deux étapes que nous décrivons dans la première partie et qui sont le choix du modèle de turbulence et le développement d'une technique de résolution numérique. Les résultats que nous présentons dans la seconde partie de ce travail sont des comparaisons entre des mesures réalisées dans des conditions réelles de propagation et notre modèle.

\section{LA THEORIE}

Notre modèle de représentation de la turbulence repose sur une idée de Kraichnan [1], reprise et développée pour les besoins de l'acoustique par M.J. Karweit et al.[2] et Y. Hugon-Jeannin [3]. 
Il s'agit d'une description statistique qui consiste, dans l'hypothèse de turbulence gelée de Taylor, à imaginer la turbulence, supposée homogène et isotrope, comme un ensemble de réalisations indépendantes du champ d'indice. Dans chacune d'elle, est propagée une onde acoustique et les variations du champ de pression sont enregistrées. Nous avons accès, ainsi, à toutes les grandeurs statistiques qui caractérisent ce dernier.

Si $\vec{n}(\vec{x})$ et $\mu(\vec{x})$ sont respectivement et pour chaque réalisation l'indice de réfraction moyen et les fluctuations d'indice au point $\vec{x}$, l'indice du milieu s'écrit en $\vec{x}: n=\bar{n}+\mu$

Le calcul de $\mu(\vec{x})$ pour chaque réalisation nécessite de "passer" dans l'espace de Fourier, soit

$$
\mu(\vec{x})=\int_{R^{3}} \tilde{\mu}(\vec{K}) \exp (i \vec{K} \vec{x}) d \vec{K}
$$

L'originalité de la méthode consiste à écrire cette intégrale comme la superposition de $N_{m}$ modes de Fourier aléatoires

$$
\mu(\vec{x})=\sum_{i=1}^{N_{m}}\left|\tilde{\mu}\left(K_{i}\right)\right| \cos \left(\vec{K}_{i} \vec{x}+\Psi_{i}\right)
$$

$\left(\vec{K}_{i} \cdot \vec{x}\right)$ est une variable aléatoire qui donne l'orientation du vecteur d'onde, un tirage de cette variable dans l'intervalle $[0,2 \pi]$ assure l'isotropie de la turbulence. $\Psi_{i}$ est également une variable tirée aléatoirement dans l'intervalle $[0,2 \pi]$ de sorte que la turbulence est homogène. $\left|\tilde{\mu}\left(K_{i}\right)\right|$ est une variable déterministe obtenue après discrétisation du spectre d'énergie $G(K)$ qui s'écrit pour une turbulence décrite par une fonction de corrélation gaussienne

$$
G(K)=\frac{\left\langle\mu^{2}\right\rangle}{2} K L^{2} \exp \left(-\frac{K^{2} L^{2}}{4}\right)
$$

En ce qui concerne l'aspect propagation du son, nous nous sommes orientés vers une technique de discrétisation de l'équation parabolique récemment développée par Collins [4] en acoustique sousmarine: le Split Step Padé. Il s'agit d'une méthode mixte entre la méthode Split Step Fourier et la méthode de Différences Finies Implicites.

Soit l'équation parabolique sur l'enveloppe $u$ du champ de pression :

$$
\frac{\partial u}{\partial r}=i k_{0}(Q-1) u \quad ; \quad Q^{2}=n^{2}+\frac{1}{k_{0}^{2}} \frac{\partial^{2}}{\partial z^{2}}
$$

La méthode Split Step Padé consiste à écrire la solution formelle de l'équation 4 sous la forme

$$
u\left(r_{0}+\Delta r, z\right)=u\left(r_{0}, z\right) \exp \left[i k_{0} \Delta r(Q-1)\right]
$$

puis à approximer l'opérateur $\exp \left[i k_{0} \Delta r(Q-1)\right]$ par une fraction rationnelle d'opérateurs d'ordre 2. Il s'en suit un système matriciel du type $A^{n+1} u^{n+1}=B^{n} u^{n}$, où $A^{n}$ et $B^{n}$ sont des matrices pentadiagonales. Ce système est résolu par une technique de décomposition LU. La méthode Split Step Padé bénéficie des avantages des méthodes de Split Step Fourier et de Différences Finies : rapidité, traitement du sol à impédance et parallélisation.

\section{COMPARAISON AVEC DES MESURES EN EXTERIEUR}

\subsection{Propagation au-dessus d'un sol rigide}

Les mesures de Daigle [5] montrent que les niveaux acoustiques dans les creux d'interférence, dus à la présence d'un sol rigide, sont supérieurs aux niveaux donnés par les théories déterministes. Ce 
phénomène de lissage des interférences est lié à la décorrélation exercée sur l'onde par les inhomogénéités aléatoires de la turbulence et est parfaitement reproduit par notre modèle. Ce dernier permet de dépasser le simple calcul des niveaux moyens par la caractérisation des fluctuations du champ. Nous avons représenté sur la figure 1, deux nuages de points obtenus pour une source et un récepteur placé à $1,2 \mathrm{~m}$ au-dessus du sol et séparés l'un de l'autre de $15 \mathrm{~m}$. $L$, l'échelle de corrélation de la turbulence vaut $1,1 \mathrm{~m},\left\langle\mu^{2}\right\rangle$, la variance des fluctuations d'indice, vaut $7,7 \times 10^{-6} . G(K)$ est discrétisé par soixante modes dans l'intervalle $[0,1 ; 6]$. Chaque point du nuage représente dans le plan complexe, la partie réelle et la partie imaginaire du champ rapporté à sa valeur moyenne pour une réalisation. Ces diagrammes permettent de mettre en évidence l'existence de fluctuations de phase et/ou d'amplitude en présence de turbulence.
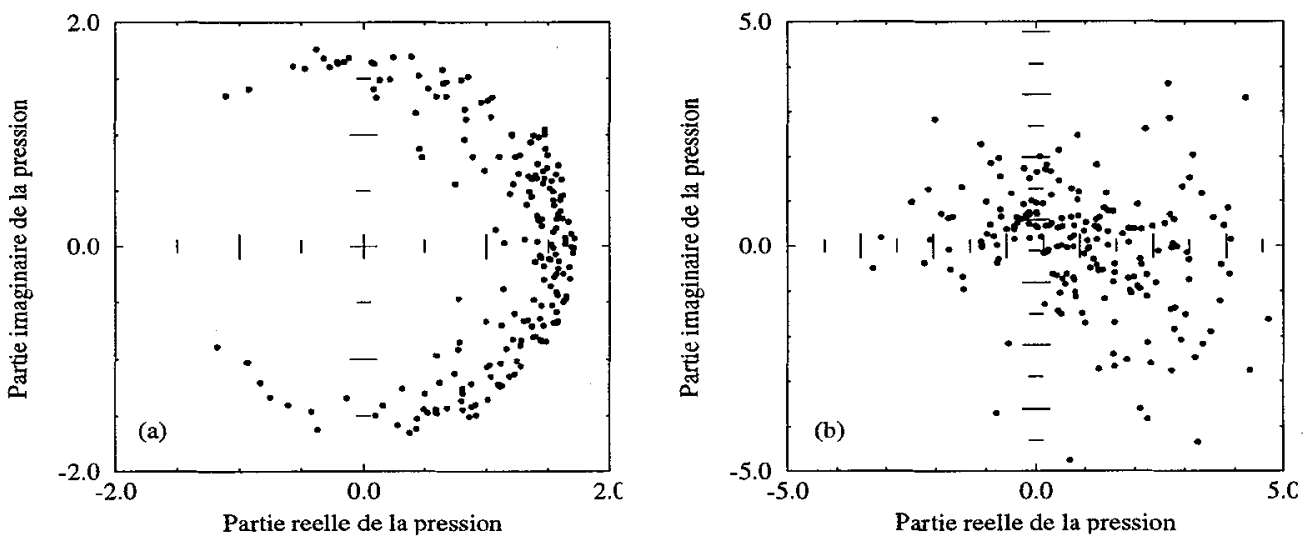

Figure 1: Nuages de points en présence d'un sol rigide, (a) sur un maximum des niveaux, (b) dans un creux d'interférence $\left(h_{s}=h_{r}=1,2 \mathrm{~m}, L=1,1 \mathrm{~m},\left\langle\mu^{2}\right\rangle=7,7 \times 10^{-6}\right)$

Le nuage de points (a) est obtenu lorsque le récepteur est sur un maximum des niveaux acoustiques. Dans ce cas, les fluctuations d'amplitude sont faibles devant les fluctuations de phase. Par contre, si le récepteur est placé dans un creux d'interférence (b), fluctuations d'amplitude et de phase sont très importantes (les échelles sont différentes sur les deux figures) correspondant à une forte perte de cohérence entre l'onde directe et l'onde réfléchie sur le sol.

\subsection{Propagation dans une zone d'ombre}

Les mesures de Wiener et Keast [6] portent sur la propagation du son dans une zone d'ombre créée par un gradient de célérité négatif. Les résultats issus de leurs campagnes expérimentales mettent en avant le rôle de la turbulence dans cette situation. Celui-ci est tout à fait différent de celui décrit précédemment, mais il n'est pas moins spectaculaire. Les inhomogénéités de la turbulence diffusent de l'énergie dans la zone d'ombre de telle sorte que le niveau relatif dans celle-ci n'est pas infiniment faible comme le prédisent les théories déterministes mais quasi-constant, se rapprochant ainsi du comportement d'une onde sphérique en espace libre. La figure 2 met en comparaison, pour les fréquences de $424 \mathrm{~Hz}$ et $848 \mathrm{~Hz}$, les mesures de Wiener et Keast, une simulation réalisée par notre modèle et un calcul déterministe. Dans les trois cas, la source et le récepteur sont placés respectivement à $3,7 \mathrm{~m}$ et $1,5 \mathrm{~m}$ au-dessus du sol. Les niveaux relatifs sont mesurés (calculés) jusqu'à une distance de $1500 \mathrm{~m}$ de la source. La turbulence est modélisée de la même façon que précédemment, l'échelle de corrélation $L$ vaut $1,1 \mathrm{~m}$ et la variance des fluctuations d'indice vaut $2 \times 10^{-6}$. Le gradient d'indice du milieu est extrapolé à partir des mesures et s'écrit : 


$$
c(z)=c_{0}-0,5 \times \log (z / d) \quad ; \quad d=6 \times 10^{-3} \mathrm{~m}
$$
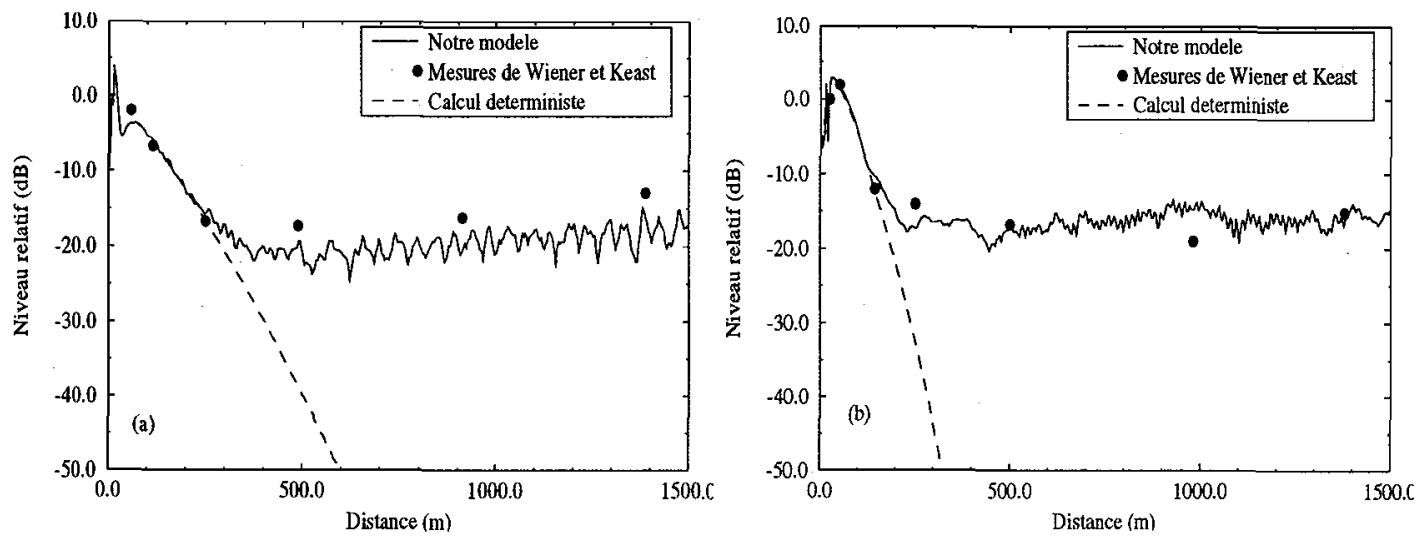

Figure 2: Niveau relatif dans une zone d'ombre: comparaison entre les mesures de Wiener et Keast, notre modèle et un calcul déterministe, (a) $f=424 \mathrm{~Hz}$, (b) $\mathrm{f}=848 \mathrm{~Hz}$ ( $h_{\mathrm{s}}=3,7 \mathrm{~m}, \mathrm{~h}_{\mathrm{r}}=1,5 \mathrm{~m}$, $L=1,1 m,\left\langle\mu^{2}\right\rangle=2 \times 10^{-6}$ )

Dans les deux situations, notre modèle traduit très bien le phénomène de diffusion par la turbulence puisque les niveaux obtenus sont extrèmement proches des mesures de Wiener et Keast. Ces figures montrent également à quel point une description déterministe est insuffisante pour ce type de problème.

\section{CONCLUSION}

Le modèle numérique que nous présentons peut s'avérer très utile pour une prédiction des propriétés du champ acoustique dans des situations de mesures délicates comme dans les zones d'ombre. Il s'agit d'un modèle statistique ce qui lui confère des avantages incontestables sur tous les modèles stochastiques classiques avec en particulier la possibilité d'obtenir des informations sur les fluctuations du signal de pression. Les résultats concernant les niveaux moyens se comparent très favorablement aux diverses mesures en extérieur et apportent une amélioration substancielle par rapport aux théories déterministes.

\section{REFERENCES}

[1] R. H. Kraichnan. The Physics of Fluids, 13(1):22-31, 1970.

[2] M. Karweit, Ph. Blanc-Benon, D. Juvé, and G. Comte-Bellot. J.Acoust.Soc.Am, 89:52-62, 1991.

[3] Y. Hugon-Jeannin. Thèse de Doctorat (92-37), Ecole centrale de Lyon, 1992.

[4] M. D. Collins and R. B. Evans. J.Acoust.Soc.Am, 91:1357-1368, 1992.

[5] G. A. Daigle, J. E. Piercy, and T. F. W. Embleton. J.Acoust.Soc.Am, 64:622-630, 1978.

[6] F. M. Wiener and D. N. Keast. J.Acoust.Soc.Am, 31:724-733, 1959. 